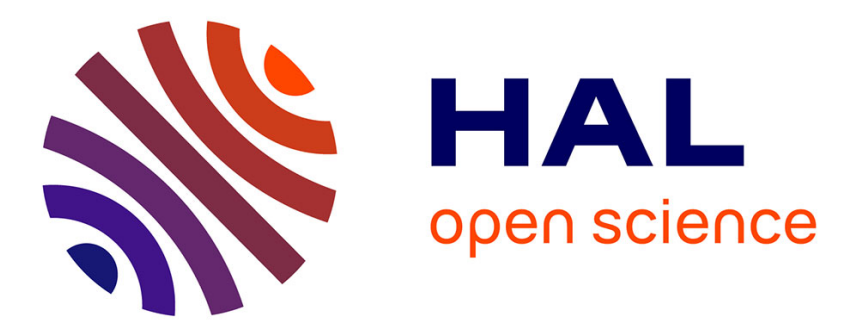

\title{
Micro-charges utiles dédiées à l'acquisition de données par drone pour l'étude des zones naturelles
}

\author{
Antoine Gademer, Loïca Avanthey, Laurent Beaudoin, Michel J. Roux,
} Jean-Paul Rudant

\section{- To cite this version:}

Antoine Gademer, Loïca Avanthey, Laurent Beaudoin, Michel J. Roux, Jean-Paul Rudant. Microcharges utiles dédiées à l'acquisition de données par drone pour l'étude des zones naturelles. Colloque scientifique francophone Drones et moyens légers aéroportés d'observation, Jun 2014, Montpellier, France. hal-01132262

\section{HAL Id: hal-01132262 https://hal.science/hal-01132262}

Submitted on 16 Mar 2015

HAL is a multi-disciplinary open access archive for the deposit and dissemination of scientific research documents, whether they are published or not. The documents may come from teaching and research institutions in France or abroad, or from public or private research centers.
L'archive ouverte pluridisciplinaire HAL, est destinée au dépôt et à la diffusion de documents scientifiques de niveau recherche, publiés ou non, émanant des établissements d'enseignement et de recherche français ou étrangers, des laboratoires publics ou privés. 


\section{Q $\frac{\text { esiea }}{A T I S}$}

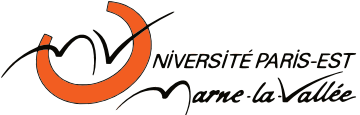

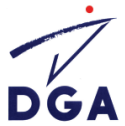

Micro-charges utiles dédiées à l'acquisition de données par drone pour l'étude des zones naturelles

A. Gademer ${ }^{\dagger} \&$ L. Avanthey ${ }^{\dagger, \star} \&$ L. Beaudoin ${ }^{\dagger} \&$ M. Roux ${ }^{\star}$ \& J.P. Rudant ${ }^{\bullet}$

$\dagger$ Laboratoire ATIS, ESIEA, www.atis-lab.fr

* Laboratoire LTCI, Institut Mines-Télécom Télécom Paristech

- Laboratoire ESYCOM, Université de Marne-la-Vallée

L'analyse des besoins particuliers de la télédétection basse altitude dans le contexte de la cartographie des milieux naturels nous a conduit à développer des capteurs dédiés innovants. Nous présentons ici un capteur modulaire tri-caméras ainsi que ses données et le retour d'expérience issus de nos missions terrains. Enfin, nous présentons notre nouveau capteur : un banc stéréoscopique amphibie.

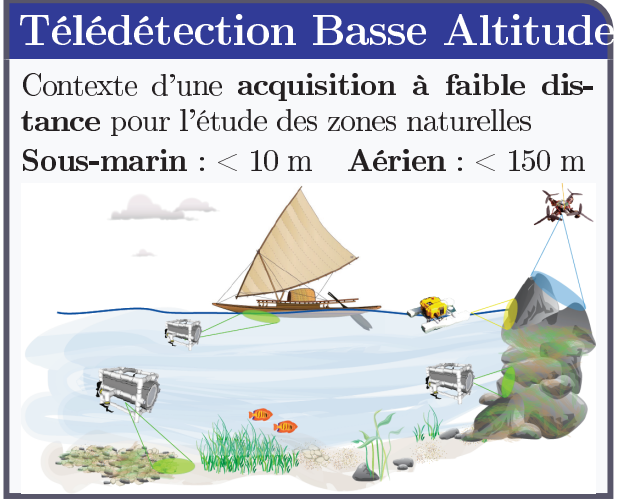

Taille de l'échantillon au sol adapté aux individus

La cartographie à l'échelle des individus permet de réaliser des analyses plus fines.

Or, pour identifier un objet, il doit être représenté au minimum par $\mathbf{4}$ à $\mathbf{9}$ pixels.

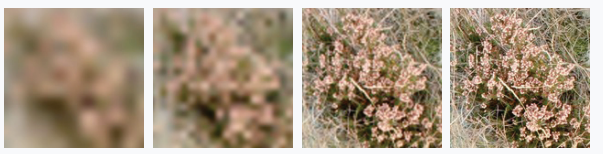

\section{Capteur tri-caméras (2009)}

- Encombrement réduit : $22 \mathrm{~cm}, 600 \mathrm{~g}$

- Simultanés

- Contrôle électronique de l'allumage,

du déclenchement et de la datation à la milliseconde des images

- Modularité : orientation au nadir ou oblique de chacun des appareils

Retour d'expérience

Le capteur tri-caméras est simultané mais pas synchronisé. On observe sur les cartes de disparité des trous liés aux bruyères qui ont bougé entre les deux images.



Besoin d'améliorer la synchronisation !

\section{Données du capteur tri-caméras et utilisation}

Les vues au nadir permettent de produire Les couples stéreoscopiques pris au nadir des mosaïques d'images sur lesquelles il permettent de reconstruire la scène en trois est possible par exemple de mesurer l'occupation des sols

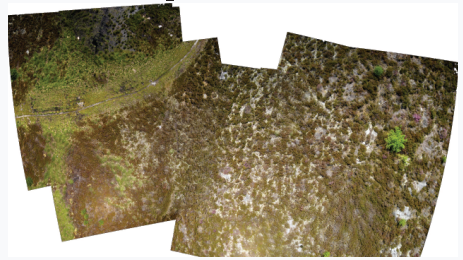

dimensions et offrent la possibilité

d'effectuer des mesures dendrométriques

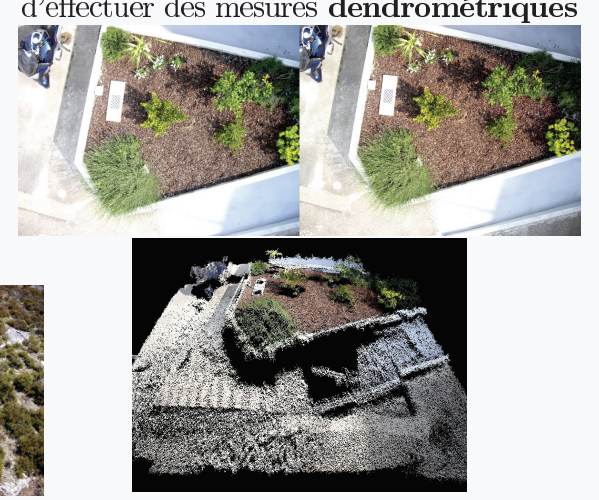

Les prises de vues obliques simultanées facilitent l'interprétation visuelle et l'estimation des hauteurs individus

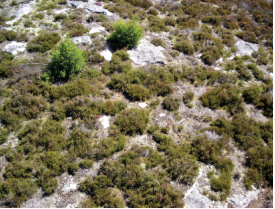

\begin{tabular}{l} 
Évolution du capteur \\
\begin{tabular}{|l|c|c|c|}
\hline Caractéristique & Tri-cam. & Banc uEye & Banc stéréo uEye (2013) \\
\hline \hline Résolution & $\mathbf{1 2 \mathrm { Mpix }}$ & $2 \mathrm{Mpix}$ \\
Datation & $0.02 \mathrm{~s}^{*}$ & $0.001 \mathrm{~s}$ \\
Synchronisation & $0.01 \mathrm{~s} *$ & $0.01 \mathrm{~s}$ \\
Traitement & variable & constant \\
Fréquence & $\mathbf{0 . 6 \mathrm { Hz }}$ & $\mathbf{1} \mathrm{Hz}$ \\
Contrôle & $*$ Hack & Ad. hoc. \\
Stockage & SDHC & Ordinateur \\
Amphibie & Non & Oui \\
\hline
\end{tabular} \\
\hline
\end{tabular}

Données du banc stéréoscopique : premiers résultats prometteurs
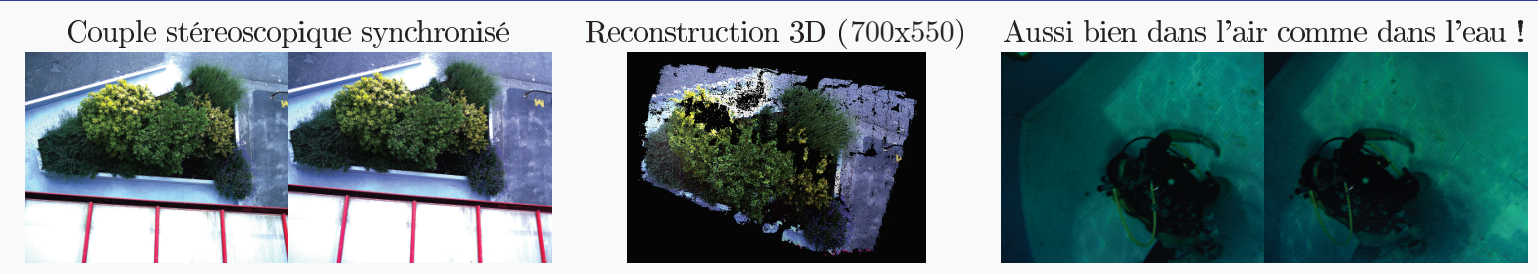

En mer

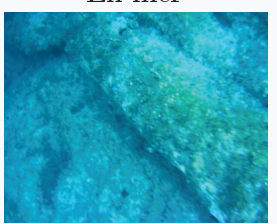

